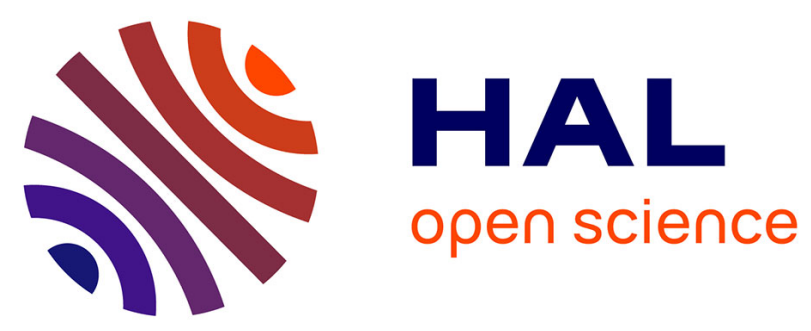

\title{
Investigation of spectral gain narrowing in quantum cascade lasers using terahertz time domain spectroscopy
}

Nathan Jukam, Sukhdeep Dhillon, Dimitri Oustinov, Zhen-Yu Zhao, Sophie

Hameau, Jérôme Tignon, Stefano Barbieri, Angela Vasanelli, Pascal Filloux, Carlo Sirtori, et al.

\section{To cite this version:}

Nathan Jukam, Sukhdeep Dhillon, Dimitri Oustinov, Zhen-Yu Zhao, Sophie Hameau, et al.. Investigation of spectral gain narrowing in quantum cascade lasers using terahertz time domain spectroscopy. Applied Physics Letters, 2008, 93, pp.101115. hal-00288345

\section{HAL Id: hal-00288345 \\ https://hal.science/hal-00288345}

Submitted on 16 Jun 2008

HAL is a multi-disciplinary open access archive for the deposit and dissemination of scientific research documents, whether they are published or not. The documents may come from teaching and research institutions in France or abroad, or from public or private research centers.
L'archive ouverte pluridisciplinaire HAL, est destinée au dépôt et à la diffusion de documents scientifiques de niveau recherche, publiés ou non, émanant des établissements d'enseignement et de recherche français ou étrangers, des laboratoires publics ou privés. 


\section{Investigation of spectral gain narrowing in quantum cascade lasers using terahertz time domain spectroscopy}

N. Jukam, S. S. Dhillon, D. Oustinov, Z.-Y. Zhao, S. Hameau, and J. Tignon

Laboratoire Pierre Aigrain, Ecole Normale Supérieure, 75231 Paris Cedex 05, France.

S. Barbieri, A.Vasanelli, P. Filloux, and C. Sirtori

Matériaux et Phénomènes Quantiques, Université Paris 7, 75251 Paris Cedex 05, France.

X. Marcadet

Thales Research and Technology, Domaine de Corbeville, 91404 Orsay cedex, France. 


\begin{abstract}
The spectral gain of bound-to-continuum terahertz quantum cascade lasers (QCLs) is measured as a function of current density using terahertz time-domain spectroscopy. During lasing action the full width at half maximum (FWHM) of the gain is found to monotonically decrease with increasing current density until lasing action stops at which point the FWHM reaches a minimum $(0.22 \mathrm{THz}$ for a laser operating at $2.1 \mathrm{THz})$. Bandstructure calculations show that the spectral gain narrowing is due to the alignment and misalignment of the injector with the active region as a function of the applied bias field.
\end{abstract}


Important progress on terahertz quantum cascade lasers (QCLs) has been achieved in the last few years leading to long wavelength ${ }^{1}$, high power $^{2}$, low current operation $^{3}$, and working temperatures up to $178 \mathrm{~K}^{4}$. In order to realize further improvements, a better understanding of the gain formation mechanism, and its limiting factors are needed. To this end it is necessary to perform detailed measurements of the gain including its spectral shape. Previous studies have been performed for mid-infrared QCLs. For these measurements, electro-luminescence from a non-lasing cavity is coupled into an adjacent cavity that shows laser action. ${ }^{5}$ However, in the terahertz regime such electro-luminescence based studies are difficult, because of the reduced spontaneous emission at longer wavelengths. Electro-luminescence from the laser cavity has also been used to provide information on upper state lifetimes of terahertz QCLs. ${ }^{6}$ In this case to avoid the effect of laser emission, the electro-luminescence from the laser cavity must be collected from a cleaved edge running though the middle of the laser. ${ }^{7}$ Multiple probe pulses coupled into the QCL's end facets can also be used to investigate the temporal dynamics of the gain. Coherent population transfer and gain saturation have been observed with this technique at mid-infrared frequencies. ${ }^{8,9}$ Recently, terahertz timedomain spectroscopy (TDS) has been shown to be a powerful technique to measure the gain spectra in terahertz QCLs. ${ }^{10,11,12}$ Here, a broadband terahertz probe pulse is coupled into the QCL, and the electric field of the transmitted pulses is measured using electrooptic sampling. ${ }^{13}$

In this letter terahertz TDS is used to investigate the line-width of the spectral gain as a function of current density. Two terahertz QCLs lasers with different bound-tocontinuum designs are studied. One laser emits at $2.1 \mathrm{THz}^{14}$ and the other emits at 2.9THz. ${ }^{15}$ For both devices, as the current density is increased from threshold, we observe a monotonic decrease of the full width at half maximum (FWHM) of the gain. After the 
laser reaches maximum power, this gain narrowing increases sharply, until laser action ceases. By calculating the band structure for different bias fields, we show the gain narrowing is a consequence of a misalignment of the upper state of the laser transition with the injector miniband.

The $2.1 \mathrm{THz}(2.9 \mathrm{THz})$ sample has an active region thickness of $14 \mu \mathrm{m}(12 \mu \mathrm{m})$, and a ridge width of $230 \mu \mathrm{m}(212 \mu \mathrm{m})$. Details on the design, processing, and performance can be found elsewhere. ${ }^{14,15}$ Samples were cleaved into $3 \mathrm{~mm}$ long laser bars. In order to improve heat sinking, all samples are bonded with indium to Au-coated copper holders which are then mounted onto a cold finger of a continuous flow helium cryostat.

The terahertz probe pulses are generated using an inter-digitated photoconductive antenna $^{16}$ fabricated on a semi-insulating GaAs wafer. A mode-locked femtosecond Ti:Saphire laser is used to excite the antenna. The THz probe pulses are collimated and refocused with parabolic mirrors onto the facet of the QCLs in the cryostat. To inject the terahertz radiation only inside the laser ridge, the input laser facet is abutted next to a hole with a nominal diameter of $200 \mu \mathrm{m}$ in a $50 \mu \mathrm{m}$ thick metal shim. After passing through the QCLs, the transmitted terahertz probe pulses are focused onto a $200 \mu \mathrm{m}$ thick ZnTe crystal together with a femtosecond probe beam. Electro-optic detection is used to measure the electric field of the THz probe pulses ${ }^{12}$.

A great advantage of this technique is that, the phase of the emitted QCL radiation is not locked in time with respect to the femtosecond probe beam; therefore no electrooptic signal is detected from the QCL emission. This allows investigations into the spectral gain below as well as above threshold. This would not be possible with an intensity-based detection technique, since the QCL emission impinging on the detector would be several orders of magnitude greater than the terahertz probe pulses. 
The QCL is modulated with $10 \mu$ s electrical pulses with a period of $40 \mu \mathrm{s}$. The inter-digitated antenna is modulated with $10 \mu$ s electrical pulses with a period of $20 \mu \mathrm{s}$, such that the QCL was on and then off for every other antenna pulse. This allows the difference between the THz electric fields with the QCL on and off (the difference field), to be measured. The gain spectra can then be derived using the difference field and a reference field. ${ }^{12}$

Figure 1a shows the transmitted difference field for various current densities for the 2.1THz QCL. Oscillations of the electric field are observed, that are due to the amplification of the input pulse at the QCL gain. At the threshold current $\left(\mathrm{J}_{\mathrm{th}}=89 \mathrm{~A} / \mathrm{cm}^{2}\right)$ the field oscillations die out quickly. However as the current density (and hence the bias field) is increased more and longer lasting oscillations are observed. At the laser shut-off current $\left(136 \mathrm{~A} / \mathrm{cm}^{2}\right)$, the field oscillations last almost twice as long as at threshold, indicating a reduction in the spectral width. The gain spectra of the $2.1 \mathrm{THz}$ device is shown in figure $1 \mathrm{~b}$, clearly showing peak gain at $2.1 \mathrm{THz}$ i.e. at the emission frequency of the QCL. As the applied bias is increased the linewidth of the main peak at $2.1 \mathrm{THz}$ decreases. This can also be seen in the inset of figure 1b) which shows the Fourier transform of the difference field. Besides the main peak at $2.1 \mathrm{THz}$, a shoulder near 1.6$1.7 \mathrm{THz}$ is present. It appears to be an artifact of a small dip in the reference pulse near 1.6THz. A broad absorption feature for frequencies greater then $2.5 \mathrm{THz}$ is also present.

Figure 2a shows the V-I and L-I curves of the $2.1 \mathrm{THz}$ laser. Below, figure $2 \mathrm{~b}$ shows the gain at the laser frequency and the FWHM of the gain as a function of current density. Below threshold the gain of the laser increases sharply after $60 \mathrm{~A} / \mathrm{cm}^{2}$ as the structure begins to align. The measured gain clamps near threshold $\left(93 \mathrm{~A} / \mathrm{cm}^{2}\right)$ at $11 \mathrm{~cm}^{-1}$ due to laser action. The gain remains constant until approximately $136 \mathrm{~A} / \mathrm{cm}^{2}$ (where laser action ceases) after which it decreases rapidly. 
Regarding the FWHM, as the bandstructure aligns after $60 \mathrm{~A} / \mathrm{cm}^{2}$ the line width of the spectral gain decreases as the gain rapidly increases. Near laser threshold there appears to be a discontinuity in the derivative of FWHM with respect to current density $(\mathrm{dFWHM} / \mathrm{dJ})$ as gain clamping begins. Beyond this point the FWHM decreases at a much slower rate until the laser power saturates at $114 \mathrm{~A} / \mathrm{cm}^{2}$. As soon as the power begins to decrease, a much sharper decrease of the FWHM is observed until it reaches a minimium of $0.22 \mathrm{THz}$ at the shut-off current density of the QCL $\left(136 \mathrm{~A} / \mathrm{cm}^{2}\right)$.

Figure 3 shows V-I and L-I curves of the 2.9THz laser along with the maximum spectral gain, the gain at the lasing frequency, and the FWHM of the spectral gain. As in the case of the $2.1 \mathrm{THz}$ laser, before threshold the gain increases and the linewidth decreases, until the gain clamps near threshold $\left(104 \mathrm{~A} / \mathrm{cm}^{2}\right)$ at $7 \mathrm{~cm}^{-1}$. At which point the derivative of the FWHM with respect to current density (dFWHM/dJ) undergoes a discontinuity as seen above. After maximum output power $\left(174 \mathrm{~A} / \mathrm{cm}^{2}\right)$, the FWHM decreases at a much greater rate with current density until a minimum linewidth of $0.12 \mathrm{THz}$ is reached at the laser shut-off current of $199 \mathrm{~A} / \mathrm{cm}^{2}$.

For both the 2.1THz and 2.9THz QCLs the spectral gain width narrows during lasing action, and decreases dramatically after peak power emission. We qualitatively explain these results in terms of the alignment and misalignment of the wavefunctions with applied bias (for reasons of length only the band diagrams of the $2.1 \mathrm{THz}$ QCL will be analysed in detail). Figures $4 \mathrm{a}, \mathrm{b}$, and c show the bandstructure of the $2.1 \mathrm{THz}$ structure for bias fields of $1.5 \mathrm{kV} / \mathrm{cm}, 1.9 \mathrm{kV} / \mathrm{cm}$, and $2.3 \mathrm{kV} / \mathrm{cm}$ respectively, along with their corresponding moduli squared wave functions. The upper laser states (labeled L1,L2, and L) are red, and the miniband states are blue. The active region (where the bulk of the lasing transition's wavefunctions overlap) consists of 3 wells separated by two thin barriers. It is indicated by the horizontal arrows in figure $4 \mathrm{a}, \mathrm{b}$, and $\mathrm{c}$. The injector 
miniband states are coupled to the upper laser state via a 50nm injector well - the pink shadded region in figure $4 a, b, c$. The injector state in green (labeled i in figure $4 a, b, c)$ is mainly confined to this $50 \mathrm{~nm}$ injector well. A simplied schematic of the bandstructures (in figures $4 \mathrm{~d}$, e, and $\mathrm{f}$ ) indicates the position of the upper laser state with respect to the injector miniband for each of the bandstructures in Figure 4a, b, and c.

For low bias fields the upper laser state is aligned with the lower region of the injector miniband, as shown in figures $4 \mathrm{a}$ and $\mathrm{d}$ for a field of $1.5 \mathrm{kV} / \mathrm{cm}$. The laser state strongly couples to the closest miniband state, which forms two strongly coupled states (L1 and L2 in figure 4a.) in the active region. The injector state (labeled i) is below levels L1 and L2 in figure 4a, and is weakly coupled to them, indicating non-optimal injection efficiency.

As the applied bias is increased the injector miniband is shifted to higher energies with respect to the upper laser states. For some bias, the laser state will be aligned with the lowest energy state of the injector miniband (the injector state) as shown in figure e. In figure $4 \mathrm{~b}$ at a bias field of $1.9 \mathrm{kV} / \mathrm{cm}$, the laser state is strongly coupled to the injector state. ${ }^{17}$ Indeed it is difficult to distinguish between the injector and laser states in figure 4b. The strong coupling between the laser and injector states corresponds to resonant current injection $^{18}$ into the upper laser level (i.e. the fastest pumping rate), and the maximium emitted power from the QCL.

For bias fields greater than the optimum bias, the injector miniband moves above the upper laser state, as shown in figure $4 \mathrm{f}$. This results in a misalignment of the structure, a decrease in the injection efficiency, and hence a drop in the emitted QCL power. This is shown in figure $4 \mathrm{c}$ for a field of $2.3 \mathrm{kV} / \mathrm{cm}$, where the laser state is below the injector state, and the injector and laser states have become uncoupled. Indeed, the oscillator strength between the injector and the lower laser state (labeled $\mathrm{g}$ in figure $4 \mathrm{a}, \mathrm{b}$, and $\mathrm{c}$ ) 
decreases from a maximum of 10 at a field of $1.9 \mathrm{kV} / \mathrm{cm}$ to less than 3 at $2.3 \mathrm{kV} / \mathrm{cm}$, while the oscillator strength between the upper and lower laser state changes from 17 at $1.9 \mathrm{kV} / \mathrm{cm}$ to 24 at $2.3 \mathrm{kV} / \mathrm{cm}$. Thus for bias fields higher then the optimal injection (i.e. maximum power), shown in figure $4 \mathrm{~b}$, a single upper laser state contributes to the gain.

Therefore the observed narrowing of the spectral gain width after maximum power (i.e. beyond $1.9 \mathrm{kV} / \mathrm{cm}$ - shown in figure $4 \mathrm{~b}$ ) is attributed to the formation of single upper laser level. This is in contrast to lower biases, where several highly coupled levels will contribute to the lasing transition. The energy splitting between the strongly coupled levels will increase the width of spectral gain vis-a-vis a single upper laser state. Bandstructure simulations of the $2.9 \mathrm{THz}$ QCL show a similar effect explaining the comparable decrease in linewidth after maximum laser emission is reached.

The broad absorption beyond $2.5 \mathrm{THz}$ for the $2.1 \mathrm{THz}$ QCL (figure 1b) could be due to transtions from the upper lasing states to the first excited miniband levels (not shown in Figure 4). These features are, however, at the limit of our measuring technique, which only has a spectral response to $\sim 3.3 \mathrm{THz}$.

In conclusion, narrowing of the gain spectrum with increasing current density has been observed above laser threshold in $2.1 \mathrm{THz}$ and $2.9 \mathrm{THz}$ bound-to-continuum quantum cascade lasers. The gain narrowing increases sharply after the maximum lasing power is reached, and the linewidth of the gain reaches a minimum when laser action ceases. The narrowing of the gain spectra is explained in term of the coupling between the upper laser state and states in the injector miniband. For low biases the upper laser state couples to several minbands states, whose energy separation augments the linewidth of the lasing transition. For higher biases, where the structure begins to misalign, the upper laser state is only weakly coupled to the injector miniband, and the laser transition arises from a single state. These results demonstrate that terahertz time domain spectroscopy, due to its 
insensitivity to the laser emission, is a powerful tool for investigating the internal bandstructure alignment of QCLs. These investigations should have an impact on future QCL designs as well as an increased understanding of the injector-laser state coupling.

\section{Acknowledgements}

This work was financially supported by the Délégation Générale pour l'Armement (DGA), Region Ile de France and the Agence Nationale pour la Recherche (ANR). The Laboratoire Pierre Aigrain of the ENS is a "Unité Mixte de Recherche Associée au CNRS UMR8551 et aux Universités Paris 6 et 7". 


\section{Figure Captions}

Figure 1: (Color online) a) Difference electric fields ( $\left.\mathrm{E}_{\mathrm{QCL} \mathrm{ON}}-\mathrm{E}_{\mathrm{QCL}} \mathrm{OFF}\right)$ of the $2.1 \mathrm{THz}$ QCL for various current densities. $89 \mathrm{~A} / \mathrm{cm}^{2}$ and $136 \mathrm{~A} / \mathrm{cm}^{2}$ correspond to the laser threshold and the laser shut-off point. b) Measured spectral gain of the $2.1 \mathrm{THz}$ QCL for various current densities. The insert shows the Fourier transform of the difference fields of figure 1a.

Figure 2: (Color online) a) V-I and L-I curves of the $2.1 \mathrm{THz}$ laser at $4.2 \mathrm{~K}$ b) Spectral gain at the laser frequency, and FWHM of the gain as a function of current density at 4.2K. The vertical dashed lines correspond, from left to right, laser threshold, peak power output and laser shut-off.

Figure 3: (Color online) a) The V-I and L-I curves of the $2.9 \mathrm{THz}$ laser at $10 \mathrm{~K}$. b) Spectral gain at the laser frequency and FWHM of the gain as a function of current density at $10 \mathrm{~K}$. The vertical dashed lines correspond, from left to right, laser threshold, peak power output and laser shut-off.

Figure 4: a, b, and c) Square modulus of the wave functions, with the injector well (shaded pink) for biases of $1.5,1.9$ and $2.3 \mathrm{kV} / \mathrm{cm}$. The upper laser states (red) are labeled L1, L2 and L. The lower laser state is labeled g. The miniband states are in blue. The injector well state (labeled i) is in green. d, e, and f) Schematic of upper laser state and injector miniband alignment corresponding to figures $4 \mathrm{a}, \mathrm{b}$, and c respectively. 


\section{References}

1) C. Walther, M. Fischer, G. Scalari, R. Terazzi, N. Hoyler and J. Faist, Appl. Phys. Lett. 91, 131122 (2007).

2) B.S. Williams, S. Kumar, Q. Hu and J.L. Reno, Elect Lett. 42, 89 (2006).

3) S. Dhillon, J. Alton, S. Barbieri and C. Sirtori, Appl. Phys. Lett. 87, 071107 (2005).

4) M. A. Belkin, J. A. Fan, S. Hormoz, F. Capasso, S.P. Khanna, M. Lachab, A. G. Davies, and E. H. Linfield, Opt. Express 16, 3242 (2008); B.S. Williams, S. Kumar, Q. Hu and J. L. Reno, Opt. Express 13, 3331 (2005).

5) S. Barbieri, C. Sirtori, H. Page, M. Beck, J. Faist and J. Nagle, IEEE J. Quant. Elect. 36, 736 (2000).

6) G. Scalari, L. Ajili, J. Faist, H. Beere, E. Linfield, D. Ritchie and G. Davies, Appl. Phys. Lett. 23, 3165 (2003).

7) R. Colombelli, F. Capasso, C. Gmachl, A. Tredicucci, A. M. Sergent, A. L. Hutchinson, D. L. Sivco and A. Y. Cho, Appl. Phys. Lett. 77, 3893 (2000).

8) F. Eickemeyer, K. Reimann, M. Woerner, and T. Elsaesser, S. Barbieri, C. Sirtori, G. Strasser, T. Muller, R. Bratschixch, and K. Unterrainer, Phys. Rev. Lett. 89, 047402 (2002).

9) H. Choi, T. B. Norris, T. Gresch, M. Girovannini, J. Faist, L. Diehl and F. Capasso, Appl. Phys. Lett 92, 122114 (2008).

10) J. Kröll, J. Darmo, S. S. Dhillon, X. Marcadet, M. Calligaro, C. Sirtori and K. Unterrainer, Nature 449, 698 (2007).

11) J. Kröll, J. Darmo, K. Unterrainer, S. S. Dhillon, C. Sirtori, X. Marcadet and M. Calligaro, Appl. Phys. Lett. 91, 161108 (2007). 
12) N. Jukam, S. S. Dhillon, Z. Y. Zhao, G. Duerr, J. Armijo, N. Sirmons, S. Hameau, S. Barbieri, P. Filloux, C. Sirtori, X. Marcadet and J. Tignon, IEEE J. Selected Topics in Quantum Electronics 14, 436 (2008).

13) Q. Wu and X. C. Zhang, Appl. Phys. Lett., 68, 1604 (1996).

14) C. Worrall, J. Alton, M. Houghton, S. Barbieri, H. E. Beere, D. A. Ritchie and C. Sirtori, Optics Express 14, 171 (2006).

15) S. Barbieri, J. Alton, H. E. Beere, J. Fowler, E. H. Linfield and D. A. Ritchie, Appl. Phys. Lett., 85, 1674 (2004).

16) A. Dreyhaupt, S. Winnerl, T. Dekorsy and M. Helm, Appl. Phys. Lett. 86, 121114 (2005).

17) The energy splitting between the upper laser state and the injector state is a local minimum at $1.9 \mathrm{kV} / \mathrm{cm}$ which indicates that maximium coupling between the states.

18) C. Sirtori, F. Capasso, J. Faist, A. L. Hutchinson, D. L. Sivco and A.Y. Cho, IEEE J. Quant. Elect. 34, 1722 (1998). 

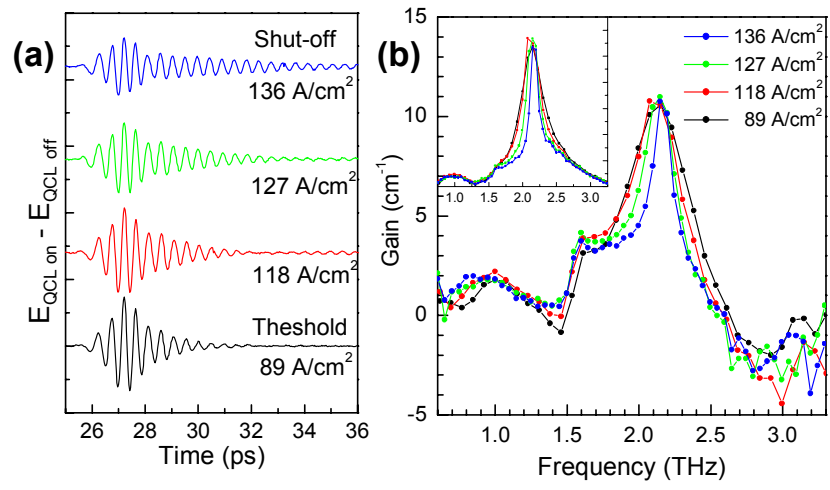


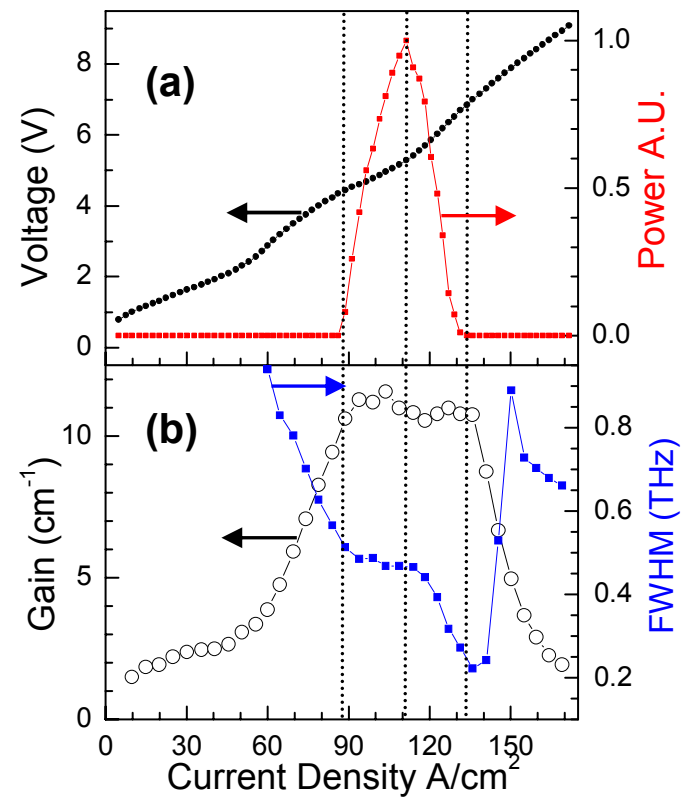




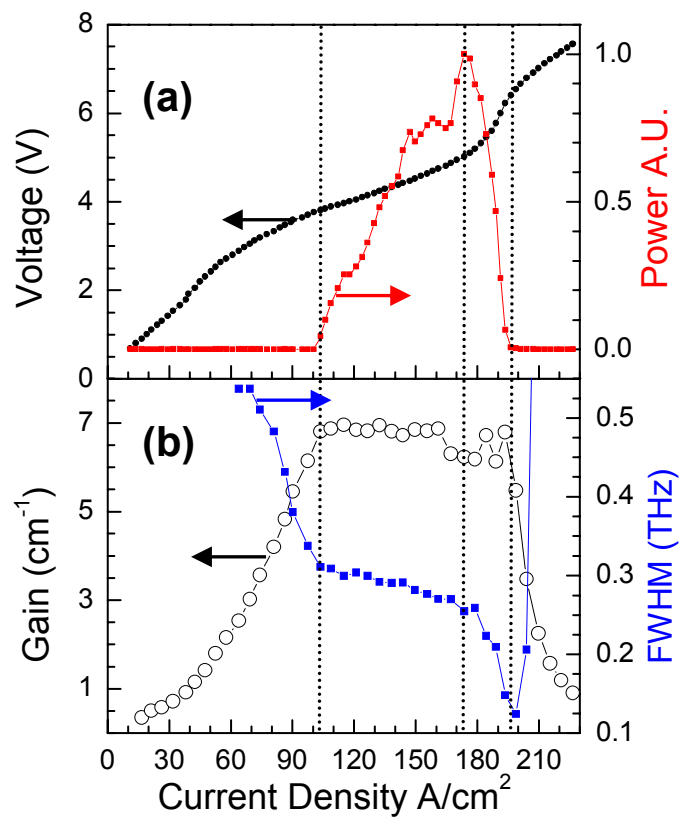




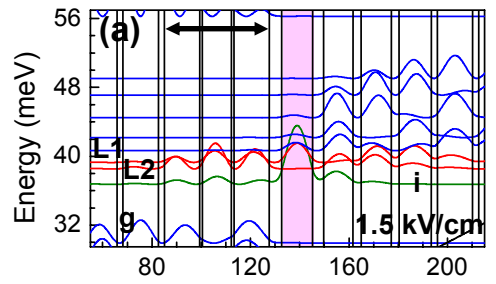

(d)

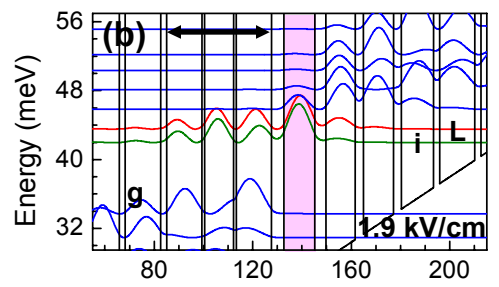

laser level Injector

miniband

miniband

(e)

miniband

miniband

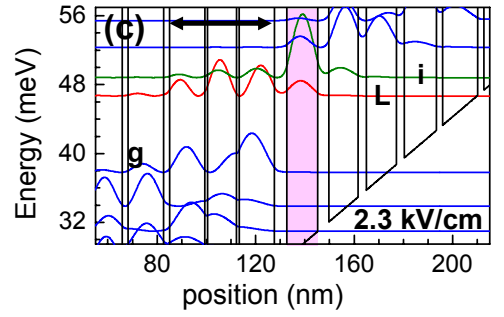

(f)

laser level

Injector

miniband

miniband 